\title{
GENERATING FUNCTIONS FOR SOME CLASSES OF UNIVALENT FUNCTIONS
}

\author{
ZDZISŁAW LEWANDOWSKI, SANFORD MILLER ${ }^{1}$ AND ELIGIUSZ ZXOTKIEWICZ
}

\begin{abstract}
Let $p(z)=e^{i \beta}+p_{1} z+p_{2} z^{2}+\cdots$ be regular in the unit disc $\Delta$ with $|\beta|<\pi / 2$, and let $\psi(u, v)$ be a continuous function defined in a domain of $\mathbf{C} \times \mathbf{C}$. With some very simple restrictions on $\psi(u, v)$ the authors prove a lemma that $\operatorname{Re} \psi\left(p(z), z p^{\prime}(z)\right)>0$ implies $\operatorname{Re} p(z)>0$. This result is then used to generate subclasses of starlike, spirallike and close-to-convex functions.
\end{abstract}

1. Introduction. In a recent paper [7] it was shown that if $f(z)=z+a_{2} z^{2}$ $+\cdots$ is regular in the unit disc $\Delta$, with $f(z) f^{\prime}(z) / z \neq 0$ in $\Delta$, and if $\alpha$ is a real number, then

$$
\begin{aligned}
\operatorname{Re}\left[(1-\alpha) \frac{z f^{\prime}(z)}{f(z)}+\alpha\left(\frac{z f^{\prime \prime}(z)}{f^{\prime}(z)}+1\right)\right] & >0, \quad z \in \Delta, \\
& \Rightarrow \operatorname{Re}\left[z f^{\prime}(z) / f(z)\right]>0, \quad z \in \Delta ;
\end{aligned}
$$

thus showing that functions $f(z)$ in the class of $\alpha$-convex functions, $\mathfrak{T}_{\alpha}$, are in fact starlike.

We can set $p(z)=z f^{\prime}(z) / f(z)$ and then $p(0)=1, p(z) \neq 0$ and we see that condition (1) is equivalent to

$$
\begin{aligned}
\operatorname{Re}\left[p(z)+\alpha \frac{z p^{\prime}(z)}{p(z)}\right] & >0, \quad z \in \Delta, \\
& \Rightarrow \operatorname{Re} p(z)>0, \quad z \in \Delta .
\end{aligned}
$$

All of the inequalities in this paper hold uniformly in the unit disc $\Delta$, and in what follows we shall omit the condition " $z \in \Delta$ ". Furthermore, if we let $\psi(u, v)=u+\alpha v / u$, then (2) becomes

$$
\operatorname{Re} \psi\left(p(z), z p^{\prime}(z)\right)>0 \Rightarrow \operatorname{Re} p(z)>0 .
$$

In this paper we prove (3) for a general class of functions $\psi(u, v)$ and then use this result to generate subclasses of starlike, spirallike and close-to-convex functions.

Received by the editors September 14, 1974.

AMS (MOS) subject classifications (1970). Primary 30A32, 34A40; Secondary 30A04, 30A20.

Key words and phrases. Functions with positive real part, Carathéodory functions, univalent functions, starlike functions, spirallike functions, close-to-convex functions.

${ }^{1}$ This work was carried out while the second author was an I.R.E.X. Scholar in Poland. 


\section{Definitions and a fundamental lemma.}

Definition 2.1. Let $u=u_{1}+u_{2} i, v=v_{1}+v_{2} i$ and let $\Psi$ be the set of functions $\psi(u, v)$ satisfying:

(a) $\psi(u, v)$ is continuous in a domain $D$ of $\mathbf{C} \times \mathbf{C}$,

(b) $(1,0) \in D$ and $\operatorname{Re} \psi(1,0)>0$,

(c) $\operatorname{Re} \psi\left(u_{2} i, v_{1}\right) \leqq 0$ when $\left(u_{2} i, v_{1}\right) \in D$ and $v_{1} \leqq-\frac{1}{2}\left(1+u_{2}^{2}\right)$.

EXAMPLES. It is easy to check that each of the following functions belong to $\Psi$.

$\psi_{1}(u, v)=u+\alpha v / u, \alpha$ real, with $D=[\mathbf{C}-\{0\}] \times \mathbf{C}$.

$\psi_{2}(u, v)=u+\alpha v, \alpha \geqq 0$, with $D=\mathbf{C} \times \mathbf{C}$.

$\psi_{3}(u, v)=u-v / u^{2}$ with $D=[\mathbf{C}-\{0\}] \times \mathbf{C}$.

$\psi_{4}(u, v)=u^{2}+v$ with $D=\mathbf{C} \times \mathbf{C}$.

$\psi_{5}(u, v)=-\ln \left(v / u^{2}+\frac{1}{2}\right)$ with $D=\left\{u|| u \mid>\frac{1}{2}\right\} \times\left\{v \| v \mid<\frac{1}{8}\right\}$.

The class $\Psi$ is closed with respect to addition, and if $\psi \in \Psi$ then $1 / \psi \in \Psi$ for perhaps a different domain, and $\alpha \psi \in \Psi$ for any $\alpha>0$.

Note that condition (c) of Definition 2.1 can be replaced by $\operatorname{Re} \psi\left(u_{2} i, v_{1}\right)$ $\leqq 0$ when $\left(u_{2} i, v_{1}\right) \in D$ and $v_{1}<0$. Though some generality is lost in considering the resulting class (for example, $\psi_{5}$ is lost) it would be much easier to work with algebraically.

We will need the following generalization of Definition 2.1 for some of our later results.

Definition 2.2. Let $b=e^{i \beta}$ where $\beta$ is real and $|\beta|<\pi / 2$. Let $u=u_{1}$ $+u_{2} i, v=v_{1}+v_{2} i$ and $\Psi_{b}$ be the set of functions $\psi(u, v)$ satisfying:

(a) $\psi(u, v)$ is continuous in a domain $D$ of $\mathbf{C} \times \mathbf{C}$,

(b) $(b, 0) \in D$ and $\operatorname{Re} \psi(b, 0)>0$,

(c) $\operatorname{Re} \psi\left(u_{2} i, v_{1}\right) \leqq 0$ when $\left(u_{2} i, v_{1}\right) \in D$ and

$$
v_{1} \leqq-\frac{1}{2}\left(1-2 u_{2} \sin \beta+u_{2}^{2}\right) / \cos \beta .
$$

Note that $-\frac{1}{2}\left(1-2 u_{2} \sin \beta+u_{2}^{2}\right) / \cos \beta<0$.

From the definitions we see that $\Psi_{1}=\Psi$. It is easy to check that $\psi_{1}, \psi_{2}, \psi_{3} \in \Psi_{b}$ for any $b$, while $\psi_{4} \in \Psi_{b}$ for $b=e^{i \beta}$ with $|\beta|<\pi / 4$, and $\psi_{5} \in \Psi_{b}$ only for $b=1$.

Definition 2.3. Let $\psi \in \Psi_{b}$ with corresponding domain $D$. We denote by $\mathscr{P}_{b}(\psi)$ those functions $p(z)=b+p_{1} z+p_{2} z^{2}+\cdots$ that are regular in $\Delta$ and satisfy:

(i) $\left(p(z), z p^{\prime}(z)\right) \in D$, and

(ii) $\operatorname{Re} \psi\left(p(z), z p^{\prime}(z)\right)>0$, when $z \in \Delta$.

The class $\mathscr{P}_{b}(\psi)$ is not empty since for any $\psi \in \Psi_{b}$ it is true that $p(z)$ $=b+p_{1} z \in \mathscr{P}_{b}(\psi)$ for $\left|p_{1}\right|$ sufficiently small (depending on $\psi$ ).

We now consider the most important result of this paper.

Lemma 2.1. If $p(z) \in \mathscr{P}_{b}(\psi)$ then $\operatorname{Re} p(z)>0$.

In other words the theorem states that if $\psi \in \Psi_{b}$, with corresponding domain $D$, and if $\left(p(z), z p^{\prime}(z)\right) \in D$ then

$$
\operatorname{Re} \psi\left(p(z), z p^{\prime}(z)\right)>0 \Rightarrow \operatorname{Re} p(z)>0 .
$$

Proof. Since $p(z)=e^{i \beta}+p_{1} z+p_{2} z^{2}+\cdots$ is regular in $\Delta$, if we set 


$$
p(z)=\frac{1+w(z)}{1-w(z)} \cos \beta+i \sin \beta,
$$

then $w(0)=0, w(z) \neq 1$ and $w(z)$ is a meromorphic function in $\Delta$. We will show that $|w(z)|<1$ for $z \in \Delta$ which implies $\operatorname{Re} p(z)>0$. Suppose that $z_{0}=r_{0} e^{i \theta_{0}}$ is a point of $\Delta$ such that $\max _{|z| \leqq r_{0}}|w(z)|=\left|w\left(z_{0}\right)\right|=1$. At such a point, by using a result of I. S. Jack [3, Lemma 1] we have

$$
z_{0} w^{\prime}\left(z_{0}\right)=\rho w\left(z_{0}\right)
$$

where $\rho \geqq 1$.

Since $\left|w\left(z_{0}\right)\right|=1$ and $w\left(z_{0}\right) \neq 1$ we must have

$$
\left(1+w\left(z_{0}\right)\right) /\left(1-w\left(z_{0}\right)\right)=A i,
$$

where $A$ is real, and thus from (5) we obtain

$$
p\left(z_{0}\right)=[A \cos \beta+\sin \beta] i \equiv e i .
$$

Differentiating (5) yields $z p^{\prime}(z)=2 z w^{\prime}(z) \cos \beta /(1-w(z))^{2}$, and thus by using (6), (7) and (8) we obtain

$$
z_{0} p^{\prime}\left(z_{0}\right)=-\frac{\rho}{2}\left(A^{2}+1\right) \cos \beta=-\frac{\rho}{2} \frac{1-2 e \sin \beta+e^{2}}{\cos \beta} \equiv d .
$$

Hence at $z=z_{0}$ we have $\operatorname{Re} \psi\left(p\left(z_{0}\right), z_{0} p^{\prime}\left(z_{0}\right)\right)=\operatorname{Re} \psi(e i, d)$ where $e$ and $d$ are real and $d \leqq-\frac{1}{2}\left(1-2 e \sin \beta+e^{2}\right) / \cos \beta$. Since $\psi \in \Psi_{b}$, by (c) of Definition 2.2 we must have $\operatorname{Re} \psi\left(p\left(z_{0}\right), z_{0} p^{\prime}\left(z_{0}\right)\right) \leqq 0$, which is a contradiction of the fact that $p(z) \in \mathscr{P}_{b}(\psi)$. Hence $|w(z)|<1$ and $\operatorname{Re} p(z)>0$ for $z$ $\in \Delta$.

Remarks. (i) In the special case $b=1$, the lemma shows that $\mathscr{P}_{1}(\psi)$ is a subset of $\mathscr{P}$, the class of Carathéodory functions

(ii) If we apply the lemma to the example $\psi_{1}$ we obtain implication (2). By applying the lemma to $\psi_{2}$ and $\psi_{3}$ we obtain

$$
\operatorname{Re}\left[p(z)+\alpha z p^{\prime}(z)\right]>0, \quad \text { with } \alpha \geqq 0, \quad \Rightarrow \operatorname{Re} p(z)>0,
$$

and

$$
\operatorname{Re}\left[p(z)-z p^{\prime}(z) / p^{2}(z)\right]>0, \quad \text { with } p(z) \neq 0, \quad \Rightarrow \operatorname{Re} p(z)>0 .
$$

Ẃe see that each $\psi \in \Psi_{b}$ can be used to generate a subclass of the set of regular functions with positive real part.

Our final result of this section deals with the relationship between the coefficients of any $p(z) \in \mathscr{P}_{b}(\psi)$ and its generating function $\psi$.

THEOREM 2.1. If $p(z)=b+p_{1} z+p_{2} z^{2}+\cdots \in \mathscr{P}_{b}(\psi)$ and $\psi(u, v)$ is a holomorphic function in its domain $D$ of $\mathbf{C} \times \mathbf{C}$, then

$$
\begin{gathered}
p_{1}\left[\psi_{1}(b, 0)+\psi_{2}(b, 0)\right]=q_{1}, \\
p_{1}^{2}\left[\psi_{11}(b, 0)+2 \psi_{12}(b, 0)+\psi_{22}(b, 0)\right] \\
+2 p_{2}\left[\psi_{1}(b, 0)+2 \psi_{2}(b, 0)\right]=2 q_{2}
\end{gathered}
$$

where $\left|q_{1}\right|,\left|q_{2}\right| \leqq 2 \operatorname{Re} \psi(b, 0)$. 
Proof. Since $\psi\left(p(z), z p^{\prime}(z)\right)$ is a regular function in $\Delta$, it has a Taylor expansion of the form

$$
\psi\left(p(z), z p^{\prime}(z)\right)=q_{0}+q_{1} z+q_{2} z^{2}+\cdots
$$

valid in $\Delta$. Since $\operatorname{Re} \psi\left(p(z), z p^{\prime}(z)\right)>0$ we must have $\left|q_{1}\right|,\left|q_{2}\right| \leqq 2 \operatorname{Re} q_{0}$ $=2 \operatorname{Re} \psi(b, 0)$. Comparing coefficients in (9) we obtain (i) and (ii).

This theorem enables us to obtain coefficient-bounds very easily without resorting to tedious series methods. For example, applying the theorem to $\psi_{5}$ we quickly obtain $\left|p_{1}\right| \leqslant \ln 2$.

3. Starlike functions. Let $f(z)=z+a_{2} z^{2}+\cdots$ be regular in $\Delta$. If $\operatorname{Re} z f^{\prime}(z) / f(z)>0$ for $z \in \Delta$ then $f(z)$ is univalent and is said to be a starlike function. We denote the class of such functions by $S^{*}$. In this section we will use our principal lemma to generate subclasses of $S^{*}$ and to extend some results of S. D. Bernardi and R. J. Libera concerning starlikeness of solutions of certain differential equations.

Definition 3.1. Let $\phi(u, v)$ be any continuous function defined on a domain $D$ of $\mathbf{C} \times \mathbf{C}$. We denote by $\delta(\phi)$ those functions $f(z)=z+a_{2} z^{2}+\cdots$ that are regular in $\Delta$ with $f(z) f^{\prime}(z) / z \neq 0$, such that

(i) $\left(z f^{\prime}(z) / f(z), z f^{\prime \prime}(z) / f^{\prime}(z)+1\right) \in D$ and

(ii) $\operatorname{Re} \phi\left(z f^{\prime}(z) / f(z), z f^{\prime \prime}(z) / f^{\prime}(z)+1\right)>0$

when $z \in \Delta$.

EXAMPLES. For the following examples which involve multivalued functions we can select an appropriate principal value.

$\phi_{1}(u, v)=u$ with $D=[\mathbf{C}-\{0\}] \times \mathbf{C}$.

$\phi_{2}(u, v)=v$ with $D=[\mathbf{C}-\{0\}] \times \mathbf{C}$.

$\phi_{3}(u, v)=(1-\alpha) u+\alpha v$, with $\alpha$ real and $D=[\mathbf{C}-\{0\}] \times \mathbf{C}$.

$\phi_{4}(u, v)=u^{1-\gamma} v^{\gamma}$, with $\gamma$ real and $D=[\mathbf{C}-\{0\}] \times[\mathbf{C}-\{0\}]$.

$\phi_{5}(u, v)=u v$ with $D=[\mathbf{C}-\{0\}] \times \mathbf{C}$.

$\phi_{6}(u, v)=-\ln \left(u / v-\frac{1}{2}\right)$ with $D=\left\{u\left|\frac{1}{2}<\right| u \mid<\frac{3}{2}\right\} \times\left\{v\left|\frac{3}{2}<\right| v \mid\right\}$.

Note that $\delta\left(\phi_{1}\right)=S^{*}, \delta\left(\phi_{2}\right)=C$, the class of convex functions, $\delta\left(\phi_{3}\right)$ $=\mathfrak{N}_{\alpha}$ and $\delta\left(\phi_{4}\right)=\mathcal{L}_{\gamma}$, the class of gamma-starlike functions [5].

We now show that by suitably restricting $\phi, \delta(\phi)$ will be a nonempty class of starlike functions.

THEOREM 3.1. Let $u=u_{1}+u_{2} i, v=v_{1}+v_{2} i$ and $\phi(u, v)$ a function satisfying:

(a) $\phi(u, v)$ is continuous in a domain $D$ of $[\mathbf{C}-\{0\}] \times \mathbf{C}$,

(b) $(1,1) \in D$ and $\operatorname{Re} \phi(1,1)>0$,

(c) $\operatorname{Re} \phi\left(u_{2} i, v_{2} i\right) \leqq 0$ when $\left(u_{2} i, v_{2} i\right) \in D$ and $v_{2} / u_{2} \geqq \frac{3}{2}\left[1+1 /\left(3 u_{2}^{2}\right)\right]$.

Then $\delta(\phi)$ is nonempty and $\delta(\phi) \subset S^{*}$.

Proof. The set $\delta(\phi)$ is nonempty since for any $\phi$ satisfying (a) and (b) it is true that $f(z)=z+a_{2} z^{2} \in \delta(\phi)$ for $\left|a_{2}\right|$ sufficiently small (depending on $\phi$ ).

If $f(z) \in \delta(\phi)$ and we set $p(z)=z f^{\prime}(z) / f(z)$ for $z \in \Delta$, then $p(z) \neq 0, p(z)$ is regular, $p(0)=1$ and

$$
\phi\left(z f^{\prime}(z) / f(z), z f^{\prime \prime}(z) / f^{\prime}(z)+1\right)=\phi\left(p(z), p(z)+z p^{\prime}(z) / p(z)\right) .
$$

Since $\phi(u, v)$ has domain $D$ in $[\mathbf{C}-\{0\}] \times \mathbf{C}$, if we set $r=r_{1}+r_{2} i, s=s_{1}$ 
$+s_{2} i$ and $\psi(r, s)=\phi(r, r+s / r)$ then by (a), (b) and (c), $\psi(r, s)$ is continuous in a domain $D_{1}=\{(u, u(v-u)) \mid(u, v) \in D\}, \operatorname{Re} \psi(1,0)>0$ and $\operatorname{Re} \psi\left(r_{2} i, s_{1}\right)$ $\leqq 0$ when $s_{1} \leqq-\frac{1}{2}\left(1+r_{2}^{2}\right)$. Hence, by Definition $2.1, \psi \in \Psi$. Since $(p(z)$, $\left.z p^{\prime}(z)\right) \in D_{1}$ and $\operatorname{Re} \psi\left(p(z), z p^{\prime}(z)\right)=\operatorname{Re} \phi\left(z f^{\prime} / f, z f^{\prime \prime} / f^{\prime}+1\right)>0$ when $z$ $\in \Delta$, by Lemma 2.1 with $b=1$ we must have $\operatorname{Re} p(z)>0$. Hence $\operatorname{Re} z f^{\prime}(z) / f(z)>0$ and $f(z) \in S^{*}$.

The theorem shows that each $\phi$ satisfying (a), (b) and (c) generates a subclass of $S^{*}$. It is easy to show that examples $\phi_{1}, \ldots, \phi_{6}$ satisfy these conditions. For $\phi=\phi_{1}, \phi_{2}, \phi_{3}$, or $\phi_{4}$ we obtain known subclasses of $S^{*}$, but as a new example consider $\phi_{5}$. For $\delta\left(\phi_{5}\right)$ we have

$$
\operatorname{Re}\left(\frac{z f^{\prime}(z)}{f(z)}\right)\left(\frac{z f^{\prime \prime}(z)}{f^{\prime}(z)}+1\right)>0 \Rightarrow f(z) \in S^{*} .
$$

Note that if $\phi$ and $\theta$ satisfy (a), (b) and (c) then so do $\phi+\theta, u \phi / v$, $-u v \phi$ and $1 / \phi$ for perhaps different domains.

The following theorem is the analogue of Theorem 2.1 and provides a very quick method for calculating some coefficient inequalities. It can easily be extended to $a_{n}$.

THEOREM 3.2. If $f(z)=z+a_{2} z^{2}+\cdots \in \delta(\phi)$ and $\phi(u, v)$ is a holomorphic function in its domain, then

$$
\begin{gathered}
a_{2}\left[\phi_{1}+2 \phi_{2}\right]=q_{1}, \\
a_{3}\left[4 \phi_{1}+12 \phi_{2}\right]-a_{2}^{2}\left[2 \phi_{1}+8 \phi_{2}-\phi_{11}-4 \phi_{12}-4 \phi_{22}\right]=2 q_{2}
\end{gathered}
$$

where $\left|q_{1}\right|,\left|q_{2}\right| \leqq 2 \operatorname{Re} \phi(1,1)$ and all partial derivatives are evaluated at $(1,1)$.

We close this section by giving an application of Theorem 3.1 to a problem of S. D. Bernardi [1] and R. J. Libera [6]. In [1] it is shown that if $g \in S^{*}$ then the solution of the differential equation

$$
c f(z)+z f^{\prime}(z)=(1+c) g(z)
$$

is also in $S^{*}$, for $c=1,2,3, \ldots$ We will show that $f(z) \in S^{*}$ for complex $c$ when $\operatorname{Re} c \geqq 0$. (By elementary methods it can be shown that (10) has a regular solution provided that $c$ is not a nonnegative integer.)

Differentiating (10) logarithmically we obtain

$$
\frac{z g^{\prime}(z)}{g(z)}=\frac{z f^{\prime}(z)}{f(z)} \frac{c+z f^{\prime \prime}(z) / f^{\prime}(z)+1}{c+z f^{\prime}(z) / f(z)} \equiv \phi\left(\frac{z f^{\prime}}{f}, \frac{z f^{\prime \prime}}{f^{\prime}}+1\right)
$$

where $\phi(u, v)=u(c+v) /(c+u)$. Since $g(z) \in S^{*}$, from (11) we obtain $\operatorname{Re} \phi\left(z f^{\prime} / f, z f^{\prime \prime} / f^{\prime}+1\right)>0$, when $z \in \Delta$, and hence from Definition 3.1 we see that $f(z) \in \delta(\phi)$. It is easy to show that $\phi$ satisfies conditions (a), (b) and (c) of Theorem 3.2 when $\operatorname{Re} c \geqq 0$, and consequently we have $f(z) \in S^{*}$.

The authors wish to thank Professor P. T. Mocanu for this interesting application.

4. Spirallike functions. Let $f(z)=z+a_{2} z^{2}+\cdots$ be regular in $\Delta$ and let $\beta$ be a real number such that $|\beta|<\pi / 2$. If $\operatorname{Re}\left[e^{i \beta} z f^{\prime}(z) / f(z)\right]>0$ for $z$ 
$\in \Delta$ then $f(z)$ is univalent [8] and is said to be $\beta$-spirallike. We denote the class of such functions by $\check{S}(\beta)$. Note that $\check{S}(0)=S^{*}$.

Definition 4.1. Let $\omega(u, v)$ be any continuous function defined on a domain $D$ of $\mathbf{C} \times \mathbf{C}$. We denote by $\check{\delta}_{\beta}(\omega),|\beta|<\pi / 2$, those functions $f(z)=z+a_{2} z^{2}$ $+\cdots$ that are regular in $\Delta$ with $f(z) f^{\prime}(z) / z \neq 0$, such that

$$
\left(e^{i \beta} z f^{\prime}(z) / f(z),\left(e^{i \beta}-1\right) z f^{\prime}(z) / f(z)+z f^{\prime \prime}(z) / f^{\prime}(z)+1\right) \in D
$$

and

$$
\operatorname{Re} \omega\left(e^{i \beta} z f^{\prime} / f,\left(e^{i \beta}-1\right) z f^{\prime} / f+z f^{\prime \prime} / f^{\prime}+1\right)>0
$$

when $z \in \Delta$. Note that $\check{S}_{0}(\omega)=\delta(\omega)$.

Our main result for generating subclasses of spirallike functions is the following theorem which is easily proved by using Lemma 2.1 .

THEOREM 4.1. Let $u=u_{1}+u_{2} i, v=v_{1}+v_{2} i,|\beta|<\pi / 2$ and $\omega(u, v)$ a function satisfying:

(a) $\omega(u, v)$ is continuous in a domain $D$ of $[\mathbf{C}-\{0\}] \times \mathbf{C}$,

(b) $\left(e^{i \beta}, e^{i \beta}\right) \in D$ and $\operatorname{Re} \omega\left(e^{i \beta}, e^{i \beta}\right)>0$,

(c) $\operatorname{Re} \omega\left(u_{2} i, v_{2} i\right) \leqq 0$ when $\left(u_{2} i, v_{2} i\right) \in D$ and

$$
v_{2} / u_{2} \geqq 1+\left(1-u_{2} \sin \beta+u_{2}^{2}\right) / 2 \cos \beta .
$$

Then $\check{\check{S}}_{\beta}(\omega)$ is nonempty and $\check{\check{S}}_{\beta}(\omega) \subset \check{S}(\beta)$.

The set $\check{S}_{\beta}(\omega)$ is not empty since $z \in \check{S}_{\beta}(\omega)$ for any $\omega$ satisfying the hypothesis. Note that although some generality is lost, the theorem is still true if we replace the inequality in (c) by the simple inequality $v_{2} / u_{2}>1$.

It is easy to check that the following functions satisfy (a), (b) and (c).

$\omega_{1}(u, v)=u+\alpha(v-u)$ with $\alpha$ real and $D=[\mathbf{C}-\{0\}] \times \mathbf{C}$,

$\omega_{2}(u, v)=u(1+v-u)$ with $D=[\mathbf{C}-\{0\}] \times \mathbf{C}$.

If we apply the theorem to $\omega_{1}$ we obtain for $\alpha$ real

$$
\operatorname{Re}\left[\left(e^{i \beta}-\alpha\right) \frac{z f^{\prime}}{f}+\alpha\left(\frac{z f^{\prime \prime}}{f^{\prime}}+1\right)\right]>0 \Rightarrow f \in \check{S}(\beta),
$$

a result discussed by Eenigenburg et al. [2].

5. Close-to-convex functions. Let $f(z)=z+a_{2} z^{2}+\cdots$ be regular in $\Delta$. If there is a function $g(z) \in C$, the class of convex functions, and a real number $\beta,|\beta|<\pi / 2$, such that $\operatorname{Re}\left[e^{i \beta} f^{\prime}(z) / g^{\prime}(z)\right]>0$ for $z \in \Delta$, then $f(z)$ is univalent [4] and is said to be close-to-convex. The class of close-to-convex functions will be denoted by $K$. In the special case when $g(z)=z$ and $\beta=0$ we will denote the class by $R$.

An immediate application of Lemma 2.1 yields the following theorem.

THEOREM 5.1. Let $\psi(u, v) \in \Psi$ with corresponding domain D. If $f(z)=z$ $+a_{2} z^{2}+\cdots$ is regular in $\Delta$ and satisfies

(i) $\left(f^{\prime}(z), z f^{\prime \prime}(z)\right) \in D$ and

(ii) $\operatorname{Re} \psi\left(f^{\prime}(z), z f^{\prime \prime}(z)\right)>0$

when $z \in \Delta$, then $f(z) \in R$. 
Applying the theorem to $\psi_{1}$ when $\alpha$ is real and $f^{\prime}(z) \neq 0$ we obtain

$$
\operatorname{Re}\left[f^{\prime}(z)+\alpha z f^{\prime \prime}(z) / f^{\prime}(z)\right]>0 \Rightarrow f(z) \in R .
$$

Similarly for $\psi_{2}$, when $\alpha \geqq 0$ we get

$$
\operatorname{Re}\left[f^{\prime}(z)+\alpha z f^{\prime \prime}(z)\right]>0 \Rightarrow f(z) \in R .
$$

A more general means of generating subclasses of close-to-convex functions comes from the following theorem, which is easily proved by using Lemma 2.1.

THEOREM 5.2. Let $u=u_{1}+u_{2} i, v=v_{1}+v_{2} i$ and $\omega(u, v)$ be a function satisfying:

(a) $\omega(u, v)$ is continuous in a domain $D$ of $[\mathbf{C}-\{0\}] \times \mathbf{C}$,

(b) $\left(e^{i \beta}, 0\right) \in D$ and $\operatorname{Re} \omega\left(e^{i \beta}, 0\right)>0$,

(c) $\operatorname{Re} \omega\left(u_{2} i, v_{2} i\right) \leqq 0$ when $\left(u_{2} i, v_{2} i\right) \in D$ and

$$
u_{2} v_{2} \geqq\left(1-u_{2} \sin \beta+u_{2}^{2}\right) / 2 \cos \beta \quad(>0) .
$$

Let $g(z) \in C$. If $f(z)=z+a_{2} z^{2}+\cdots$ is regular in $\Delta$ with $f^{\prime}(z) \neq 0$, $\left(e^{i \beta} f^{\prime}(z) / g^{\prime}(z), z f^{\prime \prime}(z) / f^{\prime}(z)-z g^{\prime \prime}(z) / g^{\prime}(z)\right) \in D, \quad$ and

$$
\operatorname{Re} \omega\left(e^{i \beta} f^{\prime} / g^{\prime}, z f^{\prime \prime} / f^{\prime}-z g^{\prime \prime} / g^{\prime}\right)>0
$$

when $z \in \Delta$ then $f(z) \in K$.

We can apply the theorem to $\omega(u, v)=u(1+v)$ and obtain

$$
\operatorname{Re}\left[e^{i \beta} \frac{f^{\prime}}{g^{\prime}}\left(1+\frac{z f^{\prime \prime}}{f^{\prime}}-\frac{z g^{\prime \prime}}{g^{\prime}}\right)\right]>0 \Rightarrow f \in K .
$$

\section{BIBLIOGRAPHY}

1. S. D. Bernardi, Convex and starlike univalent functions, Trans. Amer. Math. Soc. 135 (1969), 429-446. MR 38 \# 1243.

2. P. J. Eenigenburg, S. S. Miller, P. T. Mocanu and M. O. Reade, On a subclass of Bazilevix functions, Proc. Amer. Math. Soc. 45 (1974), 88-92.

3. I. S. Jack, Functions starlike and convex of order $\alpha$, J. London Math. Soc. (2) 3 (1971), 469-474. MR 43 \# 7611.

4. W. Kaplan, Close-to-convex schlicht functions, Michigan Math. J. 1 (1952), 169-185 (1953). MR 14, 966.

5. Z. Lewandowski, S. Miller and E. Zlotkiewicz, Gamma-starlike functions, Ann. Univ. Mariae Curie-Skłodowska 28 (1974), 32-36.

6. R. J. Libera, Some classes of regular univalent functions, Proc. Amer. Math. Soc. 16 (1965), 755-758. MR 31 \#2389.

7. S. S. Miller, P. T. Mocanu and M. O. Reade, Bazilevic functions and generalized convexity, Rev. Roumaine Math. Pures Appl. 19 (1974), 213-224. MR 49 \#3105.

8. L. Špăcek, Contribution à la théorie des fonctions univalentes, Čašopis. Pěst. Mat. 62 (1933), 12-19.

DEPARTMENT OF APPLIED MATHEMATICS, MARIA CURIE-SKKODOWSKA UNIVERSITY, 20-031, LUBLIN; POLAND

DEPARTMENT OF MATHEMATICS, STATE UNIVERSITY OF NEW YORK, BROCKPORT, NEW YORK 14420 